\title{
Bacterial Membrane Vesicles: New Therapeutic Prospectives
}

\author{
Cinzia Giagulli* \\ Section of Microbiology, Department of Molecular and Translational Medicine, University of Brescia, Brescia, Italy \\ *Corresponding Author: Cinzia Giagulli, Section of Microbiology, Department of Molecular and Translational Medicine, School of \\ Medicine, University of Brescia, Brescia, Italy.
}

Received: February 19, 2018; Published: March 01, 2018

DOI: $10.31080 /$ ASMI.2018.01.0030

\section{Abbreviations}

MV: Membrane Vesicle; TLR: Toll-Like Receptor; LPS: Lipopolysaccharide; LOS: Lipooligosaccharide; MAMPs: Microbial-Associated Molecular Patterns; PRRs: Pathogen Recognition Receptors; ClyA: Cytolysin A

In recent years the bacterial MVs have gained a strong interest and represent nowadays an active and rapidly expanding area of research for their therapeutic potential. MVs are naturally released during bacterial growth in vivo and in vitro by both pathogenic and not-pathogenic Gram-negative and Gram-positive bacteria. They can have different sizes (from $20-300 \mathrm{~nm}$ ) and a lipid membrane protects their internal cargo from nuclease and protease degradation. The cargo of MVs is represented by a variety of proteins, toxins, enzymes and nucleic acids. Lipids are the most abundant component of membranes and lipoproteins, peptidoglycan, lipoteichoic acid and LPS result to be key modulators of the host immune system.

MVs play a key role in bacterial pathogenesis. Indeed, bacteria take advantage of MVs production and release for transmission of virulence factors, communication with host immune cells, inducing inflammation and regulating host immunity defence. In particular, MVs isolated from diverse species, such as Legionella pneumophila, Clostridium difficile, Porphyromonas gingivalis, Neisseria meningitidis and Staphylococcus aureus have displayed immunological outcomes, including those mediated by TLR2 and TLR4 [1-5]. Moreover, in vitro transcriptome analysis suggested that LPS structural changes can be used to alter immunological outcome [6]. The use of a novel LOS from Bartonella quintana highlighted the potential benefits of LPS-controlled immunomodulation, since this LOS acted as a TLR4 antagonist and anti-inflammatory mediator [7]. Future research on bacterial membranes from both gram-negative and gram-positive bacteria will be useful to better define immune modulation mechanisms and new possibilities for the application of MVs in therapeutic design.

MVs naturally contain immunostimulatory ligands, known as microbial-associated molecular patterns (MAMPs), which are recognized by pathogen recognition receptors (PRRs) of immune cells. The PRRs activation induces an innate immune response, with the production of pro-inflammatory cytokines and chemokines and the recruitment of immune cells. MVs can carry MAMPs distally throughout numerous tissue types activating an effective systemic innate immune response in the host. Moreover, MVs possess also the ability to induce an adaptive immune response by inducing a long-lasting humoral and cellular immune response. Indeed, mice immunization with MVs provides protection by inducing a T-cell response including Th1, Th17, and cytotoxic T-cells [8-11].
At present, the rising of emerging multidrug resistant pathogens calls for the development of new effective vaccines. MVs from different bacteria have been explorated as vaccine candidates for their immunogenic properties. In the case of Bordetella pertussis, the MV vaccine resulted comparable or more effective than the whole cell vaccine [12-14]; in the case of Acinetobacter baumannii, mice vaccinated with MVs showed a stronger antibody response than mice vaccinated with killed bacteria or purified outer membrane complexes and without the use of adjuvants [15]. Similarly, vaccination of mice with Staphylococcus aureus MVs induced a strong cell-mediated and humoral immune response without the requirement of an adjuvant $[16,17]$. Therefore, novel MV vaccines appear more effective than traditional vaccine alternatives for the broad immune response they induce. Furthermore, the selfadjuvanting properties of MVs circumvent the need for additional adjuvants, which can have adverse side effects or poorly stimulate immune system and whose availability is limited.

The potential of MV therapies can be increased by excluding toxic component, as LPS, and by packaging specific cargos or desired epitopes into MVs. LPS detoxification can be achieved by detergent treatment or genetic manipulations to generate bacterial strains with a low toxicity variant of LPS [18]. Moreover, it is feasible that MVs from Gram-positive bacteria could be exploited as a platform for recombinant vaccine production without the need for LPS detoxification. On the other side the packaging of desired epitopes into MVs can be achieved through different methods [18]: 1) the overexpression of a recombinant outer membrane protein, which can result in increased packaging into MVs, 2) the use of a signal sequence that drives the protein of interest to the periplasmic space, where it is encapsulated into MVs, 3) the selection of an anchor protein, as the surface protein ClyA, to ensure the effective incorporation into MVs.

Novel recombinant MV vaccines, though in the early stages of development, represent a great promise for the future to combat different diseases. This approach may be more appropriate for antigens that require surface presentation to retain their native immunogenic conformation, but is limited to small proteins or peptides.

At present, even if the development of bacterial MVs as a new therapeutic prospective looks promising, MV research is still at the beginning and some issues need to be addressed before their use as therapeutic agents. In particular, verification of the specificity of MV delivery is needed to rule out toxic side effects into non-target tissues. 
In conclusion, bacterial MVs show different desirable attributes, which can be exploited for the development of novel therapies. The natural immunogenicity and self-adjuvanting capability of MVs can be used for vaccine design to induce both cell-mediated and humoral immunity. Moreover, the option to display recombinant antigens on MVs by molecular techniques gives great flexibility in vaccine design. Therefore, future studies on MV bioengineering could be important for the development of effective and safe therapies to combat different infectious, chronic and inflammatory conditions.

\section{Bibliography}

1. Jung AL., et al. "Legionella pneumophila Outer Membrane Vesicles: Isolation and Analysis of Their Pro-inflammatory Potential on Macrophages". Journal of Visualized Experiments (2017): 120.

2. Nicholas A., et al. "Clostridium difficile-derived membrane vesicles induce the expression of pro-inflammatory cytokine genes and cytotoxicity in colonic epithelial cells in vitro". Microbial Pathogenesis 107 (2017): 6-11.

3. Ho MH., et al. "Characterization of Innate Immune Responses of Human Endothelial Cells Induced by Porphyromonas gingivalis and Their Derived Outer Membrane Vesicles". Frontiers in Cellular and Infection Microbiology 6 (2016): 139.

4. Zariri A., et al. "Meningococcal Outer Membrane Vesicle Composition-Dependent Activation of the Innate Immune Response". Infection and Immunity 84.10 (2016): 30243033.

5. Jeon H., et al. "Variation among Staphylococcus aureus membrane vesicle proteomes affects cytotoxicity of host cells". Microbial Pathogenesis 93 (2016): 185-193.

6. Luan L., et al. "Comparative Transcriptome Profiles of Human Blood in Response to the Toll-like Receptor 4 Ligands Lipopolysaccharide and Monophosphoryl Lipid A". Scientific Reports 7 (2017): 40050

7. Malgorzata-Miller G., et al. "Bartonella quintana lipopolysaccharide (LPS): structure and characteristics of a potent TLR4 antagonist for in-vitro and in-vivo applications". Scientific Reports 6 (2016): 34221.

8. Chatterjee D., et al. "Vibrio cholerae 0395 outer membrane vesicles modulate intestinal epithelial cells in a NOD1 protein-dependent manner and induce dendritic cell-mediated Th2/Th17 cell responses". Journal of Biological Chemistry 288.6 (2013): 4299-4309.

9. Davitt C.J., et al. "Naturally-derived bacterial nano-particles engage diverse innate receptors, driving the activation of dendritic cells and leading to the establishment of potent adaptive immune responses". Journal of Immunology 196.1 (2016): 11-76
10. Næss LM., et al. "Human T-cell responses after vaccination with the Norwegian group B meningococcal outer membrane vesicle vaccine". Infection and Immunity 66.3 (1998): 959965

11. Rosenthal JA., et al. "Mechanistic insight into the $\mathrm{T}(\mathrm{H}) 1$-biased immune response to recombinant subunit vaccines delivered by probiotic bacteria-derived outer membrane vesicles". PLoS ONE 9.11 (2014): e112802.

12. Raeven RH., et al. "Immunoproteomic profiling of Bordetella pertussis outer membrane vesicle vaccine reveals broad and balanced humoral immunogenicity". Journal of Proteome Research 14.7 (2015): 2929-2942.

13. Bottero D., et al. "Characterization of the immune response induced by pertussis OMVs-based vaccine". Vaccine 34.28 (2016): 3303-3309.

14. Gaillard ME., et al. "Acellular pertussis vaccine based on outer membrane vesicles capable of conferring both long-lasting immunity and protection against different strain genotypes". Vaccine 32.8 (2014): 931-937.

15. Huang W., et al. "Immunization against multidrug-resistant Acinetobacter baumannii effectively protects mice in both pneumonia and sepsis models". PLOS ONE 9.6 (2014) e100727.

16. Choi SJ., et al. "Active immunization with extracellular vesicles derived from Staphylococcus aureus effectively protects against staphylococcal lung infections, mainly via Th1 cellmediated immunity". PLoS ONE 10.9 (2015): e0136021.

17. Daum RS., et al. "Progress toward a Staphylococcus aureus vaccine". Clinical Infectious Diseases 54.4 (2012): 560-567.

18. Van der Pol L., et al. "Outer membrane vesicles as platform vaccine technology”. Biotechnology Journal 10.11 (2015): 1689-1706

\section{Volume 1 Issue 4 April 2018}

(C) All rights are reserved by Cinzia Giagulli. 